\title{
A cohort study of workers exposed to formaldehyde in the British chemical industry: an update
}

\author{
M J Gardner^, B Pannett, P D Winter, A M Cruddas
}

\begin{abstract}
A cohort study of workers exposed to formaldehyde in the British chemical industry in any one of six factories has been extended after the earlier published report in 1984. A further eight years of follow up to the end of 1989 have been included for the originally reported 7660 workers first employed before 1965 , and a first follow up to the same date has been carried out for 6357 workers first employed since 1964. Extensive checking of the database has taken place including records at the factories, the MRC Environmental Epidemiology Unit, and the National Health Service Central Register. The updated findings include one death from nasal cancer compared with 1.7 expected in this number of men during the follow up period-which gives no support to the original hypothesis based on animal experimental data that formaldehyde may be a nasal carcinogen in humans. There have been no cases of nasopharyngeal cancer in the cohort compared with an estimated 1.3 expected-which gives no support to the findings in a similarly designed study in the United States of an excess of cancers of the nasopharynx associated with exposure to formaldehyde. There has been a slight excess of about $12 \%$ for lung cancer with 402 deaths compared with about 359 expected. This is similar to that found in the United States study, but higher than we reported earlier before the checking procedures and extended follow up. Further analysis gives no definitive indication of this excess of lung cancer being clearly related to formaldehyde exposure, and the increase is within that generally thought
\end{abstract}

MRC Environmental Epidemiology Unit, (University of Southampton), Southampton General Hospital, Southampton SO9 4XY

M J Gardner, B Pannett, P D Winter, A M Cruddas

^Professor Gardner died January 1993. consistent with possible confounding effects of cigarette smoking (although no data are available on this point).

(British Fournal of Industrial Medicine 1993;50:827-834)

Formaldehyde has been widely used in the manufacture of resins and plastics and in the synthesis of chemical intermediates. It has also been used, for example, as a fumigant in agriculture, in embalming fluids, in processing anatomical and pathological specimens, and in the manufacture of crease resistant garments. ${ }^{1}$

Formaldehyde is an irritant to the eyes and respiratory tract and is a contact allergen..$^{2}{ }^{3}$ It is also a known mutagen. ${ }^{4}$ Concern about the carcinogenic potential of formaldehyde followed the induction of malignant tumours of the nasal mucosa in rats exposed to 14 parts per million for up to 24 months. 56

The present study is one of several epidemiological investigations initiated because of this concern. Despite a number of publications, including our initial findings to the end of $1981^{7}$ and a large cohort study of similar workers in the United States, ${ }^{8}$ the carcinogenicity or otherwise of formaldehyde in humans remains uncertain. ${ }^{9}$ This paper reports a further eight years of follow up of the mortality experience of men who were first employed from 1920 to the end of 1964 in any one of six factories in the British chemical and plastics industry where formaldehyde had been manufactured or used, and an initial follow up of men first employed in these factories from 1965 to 1982.

\section{Methods}

The methods have been described in detail elsewhere. ${ }^{7}$ Briefly, six factories that started manufacture or use of formaldehyde from the 1920 s to the 1950s were included in the study and records of employees were made available. This paper reports on 7660 men first employed before 1965 and on 6357 men first employed after 1964-a total of 
14 017 . The number 7660 differs slightly from the 7680 reported earlier ${ }^{7}$ as a result of detailed cohort eligibility checks. Also we have carried out exhaustive enquiries into the workers' records and status since the earlier publication.

Classification of exposure to formaldehyde was on the basis of recorded titles of jobs undertaken by each of the men before 1982. In the absence of actual measurements of formaldehyde before 1970, exposures have been qualitatively classified after detailed discussions on past working conditions. These categories were then roughly transformed to quantitative values on the basis of recollections of workers about acute symptoms of irritation as follows: nil/background, under $0.1 \mathrm{ppm}$; low, 0.1 $0.5 \mathrm{ppm}$; moderate, $0.6-2.0 \mathrm{ppm}$; and high, over $2 \cdot 0 \mathrm{ppm}$. All such classifications were agreed before the mortality analysis was started. Where a man had more than one job he has been classified according to the job considered to have had the highest exposure.

The follow up for mortality and cancer registration of the men to 31 December 1989 has been through the National Health Service Central Register supplemented by the National Insurance Index. Death certificates were provided for men who had died with causes of death coded to the International Classification of Diseases (ICD) revision in use at the time of death and to the current (9th) revision..$^{10}$ Expected numbers of deaths were calculated by the person-years method with age, sex, cause, and calendar period specific death rates for England and Wales. Also, the expected numbers were adjusted for mortality in the areas in which the factories are situated by using local standardised mortality ratios (SMRs) for the years $1968-78$ and $1980-9$ for which data are available. ${ }^{1112}$ The mortality analysis covers 1 January 1941 to 31 December 1989.

\section{Results}

The results are presented separately for men first employed before and after 1 January 1965 . This is to enable comparison with our earlier report on the first group. Table 1 shows the numbers and percentages in each factory classified by estimated exposure to formaldehyde. Of the men categorised to high exposure most (86\%) were from BIP. Except at Ciba-Geigy most men had only one job.

Table 2 gives mortality from all causes of death, malignant neoplasms, and respiratory diseases. Mortality overall has been similar to that expected at national death rates, with SMRs of 103 and 95 in the two subcohorts. The first is, however, an increase from an SMR of 87 in our previous results. Such an increase has also occurred for the two particular causes of death in the table. After adjustment for local mortality the SMR among men first employed before 1965 for all cancers decreases from 114 to 106 (95\% confidence interval (95\% CI) 99 to 114 ) and for respiratory diseases from 115 to 105 (95\% CI 94 to 116 ).

Table 3 shows mortality from cancers of the nose and lung, together with adjustments for the lung to take account of local death rates. One death from nasal cancer has occurred, less than the 1.74 expected. The death took place during the extended follow up period. Mortality from lung cancer shows an increase from an SMR of 95 after local adjustment in our earlier results to $112(95 \%$ confidence interval $(95 \%$ CI) $100-124)$. Some increase was found for all factories. Among men

Table 1 Number (percentage) of men by highest category of formaldehyde exposure in any job by factory with proportion who had only one job

\begin{tabular}{|c|c|c|c|c|c|c|c|}
\hline \multirow{2}{*}{$\begin{array}{l}\text { Exposure } \\
\text { category }\end{array}$} & \multirow[b]{2}{*}{ Borden } & \multicolumn{2}{|l|}{ Synthite } & \multirow{2}{*}{$\begin{array}{l}\text { British } \\
\text { Industrial } \\
\text { Plastics } \\
(B I P)\end{array}$} & \multirow[b]{2}{*}{ Ciba-Geigy } & \multirow{2}{*}{$\begin{array}{l}\text { British } \\
\text { Petroleum } \\
\text { (BP) }\end{array}$} & \multirow[b]{2}{*}{ Total } \\
\hline & & West Bromwich & Mold & & & & \\
\hline $\begin{array}{l}\text { Nil/background } \\
\text { Low } \\
\text { Moderate } \\
\text { High } \\
\text { Unknown }\end{array}$ & $\begin{array}{rr}259 & (41) \\
140 & (22) \\
112 & (18) \\
20 & (3) \\
107 & (17)\end{array}$ & $\begin{array}{rr}36 & (10) \\
192 & (53) \\
37 & (10) \\
92 & (25) \\
9 & (3)\end{array}$ & $\begin{array}{ll}33 & (16) \\
71 & (35) \\
36 & (18) \\
42 & (21) \\
20 & (10)\end{array}$ & $\begin{array}{rr}\text { First employe (0) } \\
4 & (0) \\
241 & (7) \\
448 & (13) \\
2455 & (73) \\
213 & (6)\end{array}$ & $\begin{array}{rr}184 & (19) \\
484 & (50) \\
81 & (8) \\
81 & (8) \\
133 & (14)\end{array}$ & $\begin{array}{rr}1424 & (67) \\
700 & (33) \\
0 & (0) \\
0 & (0) \\
6 & (0)\end{array}$ & $\begin{array}{rr}1940 & (25) \\
1828 & (24) \\
714 & (9) \\
2690 & (35) \\
488 & (6)\end{array}$ \\
\hline Total & $638(100)$ & $366(100)$ & $202(100)$ & $3361(100)$ & $963(100)$ & $2130(100)$ & $7660(100)$ \\
\hline One job only (\%) & 78 & 90 & 82 & 82 & 41 & 69 & 73 \\
\hline $\begin{array}{l}\text { Nil/background } \\
\text { Low } \\
\text { Moderate } \\
\text { High } \\
\text { Unknown }\end{array}$ & $\begin{array}{rr}679 & (54) \\
236 & (19) \\
253 & (20) \\
31 & (2) \\
70 & (6)\end{array}$ & $\begin{array}{rr}31 & (8) \\
148 & (37) \\
38 & (10) \\
170 & (43) \\
9 & (2)\end{array}$ & $\begin{array}{ll}24 & (9) \\
79 & (31) \\
64 & (25) \\
63 & (25) \\
27 & (11)\end{array}$ & \begin{tabular}{rr} 
First employed af & \multicolumn{1}{c}{$(0)$} \\
99 & $(7)$ \\
214 & $(15)$ \\
962 & $(67)$ \\
155 & $(11)$
\end{tabular} & $\begin{array}{rr}964 & \\
201 & (12) \\
1086 & (65) \\
79 & (5) \\
77 & (5) \\
218 & (13)\end{array}$ & $\begin{array}{rr}997 & (74) \\
339 & (25) \\
0 & (0) \\
0 & (0) \\
8 & (1)\end{array}$ & $\begin{array}{rr}1932 & (30) \\
1987 & (31) \\
648 & (10) \\
1303 & (21) \\
487 & (8)\end{array}$ \\
\hline $\begin{array}{l}\text { Total } \\
\text { One job only (\%) }\end{array}$ & $\begin{array}{l}1269(100) \\
84\end{array}$ & $\begin{array}{l}396(100) \\
96\end{array}$ & $\begin{array}{l}257(100) \\
94\end{array}$ & $\begin{array}{l}1430(100) \\
79\end{array}$ & $\begin{array}{l}1661(100) \\
62\end{array}$ & $\begin{array}{l}1344(100) \\
83\end{array}$ & $\begin{array}{l}6357(100) \\
78\end{array}$ \\
\hline
\end{tabular}


Table 2 Mortality during 1941-1989 among men by factory

\begin{tabular}{|c|c|c|c|c|c|c|c|c|c|c|}
\hline \multirow{3}{*}{$\begin{array}{l}\text { Cause of } \\
\text { death }\end{array}$} & \multirow{3}{*}{$\begin{array}{l}\text { ICD } \\
\text { No }\end{array}$} & \multirow[b]{3}{*}{ Factory } & \multicolumn{4}{|c|}{ First employed before 1965} & \multicolumn{4}{|c|}{ First employed after 1964} \\
\hline & & & \multicolumn{2}{|c|}{ No of deaths* } & \multirow[b]{2}{*}{$S M R$} & \multirow{2}{*}{$\begin{array}{l}95 \% C I \\
\text { for } S M R\end{array}$} & \multicolumn{2}{|c|}{ No of deaths* } & \multirow[b]{2}{*}{$S M R$} & \multirow{2}{*}{$\begin{array}{l}95 \% C I \\
\text { for SMR }\end{array}$} \\
\hline & & & Observed & Expected & & & Observed & Expected & & \\
\hline \multirow[t]{5}{*}{ All causes } & \multirow[t]{5}{*}{$0-999$} & $\begin{array}{l}\text { Borden } \\
\text { Synthite }\end{array}$ & 130 & 145 & 90 & $75-106$ & 102 & $105 \cdot 4$ & 97 & 79-117 \\
\hline & & $\begin{array}{l}\text { (WB) } \\
\text { Synthite }\end{array}$ & 160 & 146 & 110 & $93-128$ & 49 & $41 \cdot 9$ & 117 & $87-155$ \\
\hline & & BIP & $\begin{array}{r}73 \\
1488\end{array}$ & $\begin{array}{r}68 \\
1366\end{array}$ & $\begin{array}{l}107 \\
109\end{array}$ & $\begin{array}{r}84-134 \\
103-115\end{array}$ & $\begin{array}{r}38 \\
102\end{array}$ & $\begin{array}{r}30 \cdot 4 \\
106 \cdot 0\end{array}$ & $\begin{array}{r}125 \\
96\end{array}$ & $\begin{array}{l}89-172 \\
78-117\end{array}$ \\
\hline & & $\begin{array}{l}\text { Ciba-Geigy } \\
\text { (from 1958) } \\
\text { BP }\end{array}$ & $\begin{array}{l}179 \\
714\end{array}$ & $\begin{array}{l}233 \\
712\end{array}$ & $\begin{array}{r}77 \\
100\end{array}$ & $\begin{array}{l}66-89 \\
93-108\end{array}$ & $\begin{array}{l}86 \\
80\end{array}$ & $\begin{array}{r}117 \cdot 0 \\
81.6\end{array}$ & $\begin{array}{l}74 \\
98\end{array}$ & $\begin{array}{l}59-91 \\
78-122\end{array}$ \\
\hline & & Total & 2744 & 2671 & 103 & $99-107$ & 457 & $482 \cdot 4$ & 95 & $86-104$ \\
\hline \multirow[t]{5}{*}{$\begin{array}{l}\text { Malignant } \\
\text { tumours }\end{array}$} & \multirow[t]{5}{*}{$140-208$} & $\begin{array}{l}\text { Borden } \\
\text { Synthite }\end{array}$ & 29 & 38 & 76 & $51-110$ & 29 & $28 \cdot 7$ & 101 & $68-145$ \\
\hline & & $\begin{array}{r}\text { (WB) } \\
\text { Synthite }\end{array}$ & 49 & 38 & 128 & $94-169$ & 10 & $11 \cdot 3$ & 89 & $43-163$ \\
\hline & & $\begin{array}{l}\text { (M) } \\
\text { BIP } \\
\text { Ciba-Geigy }\end{array}$ & $\begin{array}{r}26 \\
449\end{array}$ & $\begin{array}{r}18 \\
359\end{array}$ & $\begin{array}{l}148 \\
125\end{array}$ & $\begin{array}{r}97-217 \\
114-137\end{array}$ & $\begin{array}{l}11 \\
32\end{array}$ & $\begin{array}{r}8 \cdot 5 \\
29 \cdot 5\end{array}$ & $\begin{array}{l}130 \\
109\end{array}$ & $\begin{array}{l}65-232 \\
74-153\end{array}$ \\
\hline & & $\begin{array}{l}\text { (from 1958) } \\
\text { BP }^{-19}\end{array}$ & $\begin{array}{r}47 \\
202\end{array}$ & $\begin{array}{r}63 \\
190\end{array}$ & $\begin{array}{r}75 \\
107\end{array}$ & $\begin{array}{l}55-100 \\
92-122\end{array}$ & $\begin{array}{l}21 \\
25\end{array}$ & $\begin{array}{l}32.1 \\
21.9\end{array}$ & $\begin{array}{r}65 \\
114\end{array}$ & $\begin{array}{l}41-100 \\
74-169\end{array}$ \\
\hline & & Total & 802 & 705 & 114 & $106-122$ & 128 & $131 \cdot 9$ & 97 & $81-115$ \\
\hline \multirow[t]{5}{*}{$\begin{array}{l}\text { Respiratory } \\
\text { diseases }\end{array}$} & \multirow[t]{5}{*}{$460-519$} & $\begin{array}{l}\text { Borden } \\
\text { Synthite }\end{array}$ & 12 & 16 & 74 & $38-129$ & 5 & $8 \cdot 4$ & 59 & 19-138 \\
\hline & & $\begin{array}{r}\text { (WB) } \\
\text { Synthite }\end{array}$ & 20 & 18 & 110 & $67-170$ & 10 & $4 \cdot 0$ & 253 & $121-466$ \\
\hline & & $\begin{array}{l}\quad(M) \\
\text { BIP } \\
\text { Ciba-Geigy }\end{array}$ & $\begin{array}{r}9 \\
232\end{array}$ & $\begin{array}{r}9 \\
164\end{array}$ & $\begin{array}{l}104 \\
142\end{array}$ & $\begin{array}{r}48-198 \\
124-161\end{array}$ & $\begin{array}{l}2 \\
5\end{array}$ & $\begin{array}{l}2 \cdot 8 \\
7 \cdot 4\end{array}$ & $\begin{array}{l}71 \\
68\end{array}$ & $\begin{array}{r}9-255 \\
22-158\end{array}$ \\
\hline & & BP ${ }^{\text {(from 1958) }}$ & $\begin{array}{l}16 \\
71\end{array}$ & $\begin{array}{l}25 \\
82\end{array}$ & $\begin{array}{l}64 \\
87\end{array}$ & $\begin{array}{l}37-105 \\
68-109\end{array}$ & $\begin{array}{l}5 \\
2\end{array}$ & $\begin{array}{l}8 \cdot 6 \\
5 \cdot 4\end{array}$ & $\begin{array}{l}58 \\
37\end{array}$ & $\begin{array}{r}19-136 \\
5-134\end{array}$ \\
\hline & & Total & 360 & 314 & 115 & $103-127$ & 29 & $36 \cdot 5$ & 79 & 53-114 \\
\hline
\end{tabular}

^Expected number of deaths based on England and Wales age, sex, cause, and calendar year specific rates.

ICD No = International Classification of Diseases, 9th revision code (World Health Organisation, 1977); WB, West Bromwich; $M$ = Mold; SMR = standardised mortality ratio ((observed/expected $\times 100)$.

first employed after 1964 there was also a similar excess with an SMR of 113 (95\% CI 85-147).

Table 4 shows mortality from other specific cancers among men in all factories combined. Without local adjustment table 4 indicates raised values for cancers of the rectum (as reported earlier ${ }^{7}$ ) and stomach, but both decrease after local adjustment (rectum, SMR 113 (95\% CI 82-151); stomach, SMR $124(95 \%$ CI 101-151)) and are low in those employed after 1964. The excess of stomach cancer in the earlier subcohort is consistently found, however, in each of the six factories. There have been no deaths from cancer of the nasopharynx (expected estimated overall at 1.3), and no non-fatal cases of nasal or nasopharyngeal tumours have been reported.

Table 5 gives findings for lung cancer after adjustment for local mortality by estimated category of exposure to formaldehyde. Among the earlier group of workers there is no particular suggestion of a trend in mortality with increasing exposure. The high exposure group, however, does have the highest SMR $(124,95 \%$ CI 107-144), which is largely based on the BIP factory. Although the numbers of deaths are much smaller similar results are found in those employed after 1964.

Table 6 shows lung cancer mortality among men classified as exposed to high concentrations of formaldehyde at the BIP factory by their duration of employment and duration of follow up. There is no suggestion in the earlier employed group of any increase in risk with either factor-for duration of employment, in particular, the highest SMR is among men who worked for five to nine years. For those employed after 1964, the SMRs are based on few deaths.

Table 7 shows mortality from lung cancer among men entering employment at BIP before 1965 by cumulative dose. The methods used are as described by Acheson and colleagues. ${ }^{13}$ The first analysis uses the lower levels of the four subjectively estimated categories described earlier, whereas the second analysis replaces these with scores of $0,1,2$, and 3 for the calculation of cumulative dose. The examples shown in the table are the same as in Acheson et $\mathrm{al}^{13}$ and it can be seen that in neither case is there any suggestion of a relation between mortality from lung cancer and cumulative dose. 
Table 3 Mortality from cancers of the nose and lung during 1941-1989 among men by factory with adjustment for local rates

\begin{tabular}{|c|c|c|c|c|c|c|c|c|c|c|}
\hline \multirow{3}{*}{$\begin{array}{l}\text { Cause of } \\
\text { death }\end{array}$} & \multirow{3}{*}{$\begin{array}{l}I C D \\
\text { No }\end{array}$} & \multirow[b]{3}{*}{ Factory } & \multicolumn{4}{|c|}{ First employed before 1965} & \multicolumn{4}{|c|}{ First employed after 1964} \\
\hline & & & \multicolumn{2}{|c|}{ No of deaths* } & \multirow[b]{2}{*}{$S M R$} & \multirow{2}{*}{$\begin{array}{l}95 \% C I \\
\text { for SMR }\end{array}$} & \multicolumn{2}{|c|}{ No of deaths* } & \multirow[b]{2}{*}{$S M R$} & \multirow{2}{*}{$\begin{array}{l}95 \% C I \\
\text { for SMR }\end{array}$} \\
\hline & & & Observed & Expected & & & Observed & Expected & & \\
\hline \multirow{6}{*}{$\begin{array}{l}\text { Nasal } \\
\text { cancer }\end{array}$} & \multirow[t]{6}{*}{160} & \multirow{3}{*}{$\begin{array}{l}\text { Borden } \\
\text { Synthite } \\
\text { (WB) } \\
\text { Synthite } \\
\text { (M) }\end{array}$} & 0 & 0.08 & 0 & $0-4706$ & 0 & 0.07 & 0 & $0-5580$ \\
\hline & & & 0 & 0.08 & 0 & $0-4830$ & 0 & 0.02 & 0 & $0-15178$ \\
\hline & & & 0 & 0.04 & 0 & $0-10502$ & 0 & 0.02 & 0 & $0-21105$ \\
\hline & & \multirow{2}{*}{$\begin{array}{l}\text { Ciba-Geigy } \\
\text { (from 1958) } \\
\text { BP }\end{array}$} & 0 & $0 \cdot 73$ & 0 & $0-507$ & 0 & 0.07 & 0 & $0-5181$ \\
\hline & & & $\begin{array}{l}0 \\
1\end{array}$ & $\begin{array}{l}0.13 \\
0 \cdot 39\end{array}$ & $\begin{array}{r}0 \\
260\end{array}$ & $\begin{array}{l}0-2898 \\
7-1449\end{array}$ & $\begin{array}{l}0 \\
0\end{array}$ & $\begin{array}{l}0.08 \\
0.06\end{array}$ & $\begin{array}{l}0 \\
0\end{array}$ & $\begin{array}{l}0-4852 \\
0-6676\end{array}$ \\
\hline & & Total & 1 & 1.43 & 70 & $2-390$ & 0 & 0.31 & 0 & $0-1189$ \\
\hline \multirow[t]{5}{*}{ Lung cancer } & \multirow[t]{5}{*}{$162-4$} & \multirow{2}{*}{$\begin{array}{l}\text { Borden } \\
\text { Synthite } \\
\text { (WB) } \\
\text { Synthite }\end{array}$} & 12 & 15 & 81 & $42-142$ & 14 & $10 \cdot 4$ & 135 & $74-227$ \\
\hline & & & 21 & 16 & 134 & $83-205$ & 4 & $4 \cdot 2$ & 96 & $26-245$ \\
\hline & & BIP & $\begin{array}{r}10 \\
216\end{array}$ & $\begin{array}{r}7 \\
145\end{array}$ & $\begin{array}{l}143 \\
149\end{array}$ & $\begin{array}{r}69-263 \\
130-170\end{array}$ & $\begin{array}{r}5 \\
15\end{array}$ & $\begin{array}{r}3.3 \\
10 \cdot 7\end{array}$ & $\begin{array}{l}152 \\
141\end{array}$ & $\begin{array}{l}49-354 \\
79-232\end{array}$ \\
\hline & & $\begin{array}{l}\text { Ciba-Geigy } \\
\text { (from 1958) } \\
\text { BP }\end{array}$ & $\begin{array}{l}15 \\
74\end{array}$ & $\begin{array}{l}25 \\
76\end{array}$ & $\begin{array}{l}61 \\
97\end{array}$ & $\begin{array}{l}34-101 \\
76-122\end{array}$ & $\begin{array}{l}\mathbf{8} \\
\mathbf{8}\end{array}$ & $\begin{array}{r}11 \cdot 5 \\
7 \cdot 5\end{array}$ & $\begin{array}{r}69 \\
106\end{array}$ & $\begin{array}{l}30-137 \\
46-209\end{array}$ \\
\hline & & Total & 348 & 284 & 123 & $110-136$ & 54 & $47 \cdot 6$ & 114 & $85-148$ \\
\hline \multirow{5}{*}{$\begin{array}{r}\text { Lung cancer } \\
\text { (adjusted) }\end{array}$} & \multirow[t]{5}{*}{$162-4$} & \multirow{3}{*}{$\begin{array}{l}\text { Borden } \\
\text { Synthite } \\
\text { (WB) } \\
\text { Synthite } \\
\text { (M) } \\
\text { BIP }\end{array}$} & 12 & 13 & 90 & $46-157$ & 14 & $9 \cdot 2$ & 152 & $83-254$ \\
\hline & & & 21 & 20 & 104 & $64-158$ & 4 & $5 \cdot 4$ & 74 & $20-190$ \\
\hline & & & $\begin{array}{r}10 \\
216\end{array}$ & $\begin{array}{r}8 \\
179\end{array}$ & $\begin{array}{l}121 \\
121\end{array}$ & $\begin{array}{r}58-222 \\
105-138\end{array}$ & $\begin{array}{r}5 \\
15\end{array}$ & $\begin{array}{r}3 \cdot 6 \\
13 \cdot 4\end{array}$ & $\begin{array}{l}138 \\
112\end{array}$ & $\begin{array}{l}45-323 \\
62-184\end{array}$ \\
\hline & & \multirow{2}{*}{$\begin{array}{l}\text { Ciba-Geigy } \\
\text { (from 1958) } \\
\text { BP } \\
\text { Total }\end{array}$} & $\begin{array}{l}15 \\
74\end{array}$ & $\begin{array}{l}20 \\
71\end{array}$ & $\begin{array}{r}74 \\
105\end{array}$ & $\begin{array}{l}42-123 \\
82-132\end{array}$ & $\begin{array}{l}8 \\
8\end{array}$ & $\begin{array}{l}9 \cdot 1 \\
7 \cdot 0\end{array}$ & $\begin{array}{r}88 \\
115\end{array}$ & $\begin{array}{l}38-172 \\
50-227\end{array}$ \\
\hline & & & 348 & 311 & 112 & $100-124$ & 54 & $47 \cdot 8$ & 113 & $85-147$ \\
\hline
\end{tabular}

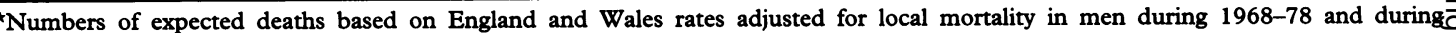
1980-89. Other explanations as in table 2.

Table 4 Mortality from specified malignant neoplasms during 1941-1989 among men in all factories combined

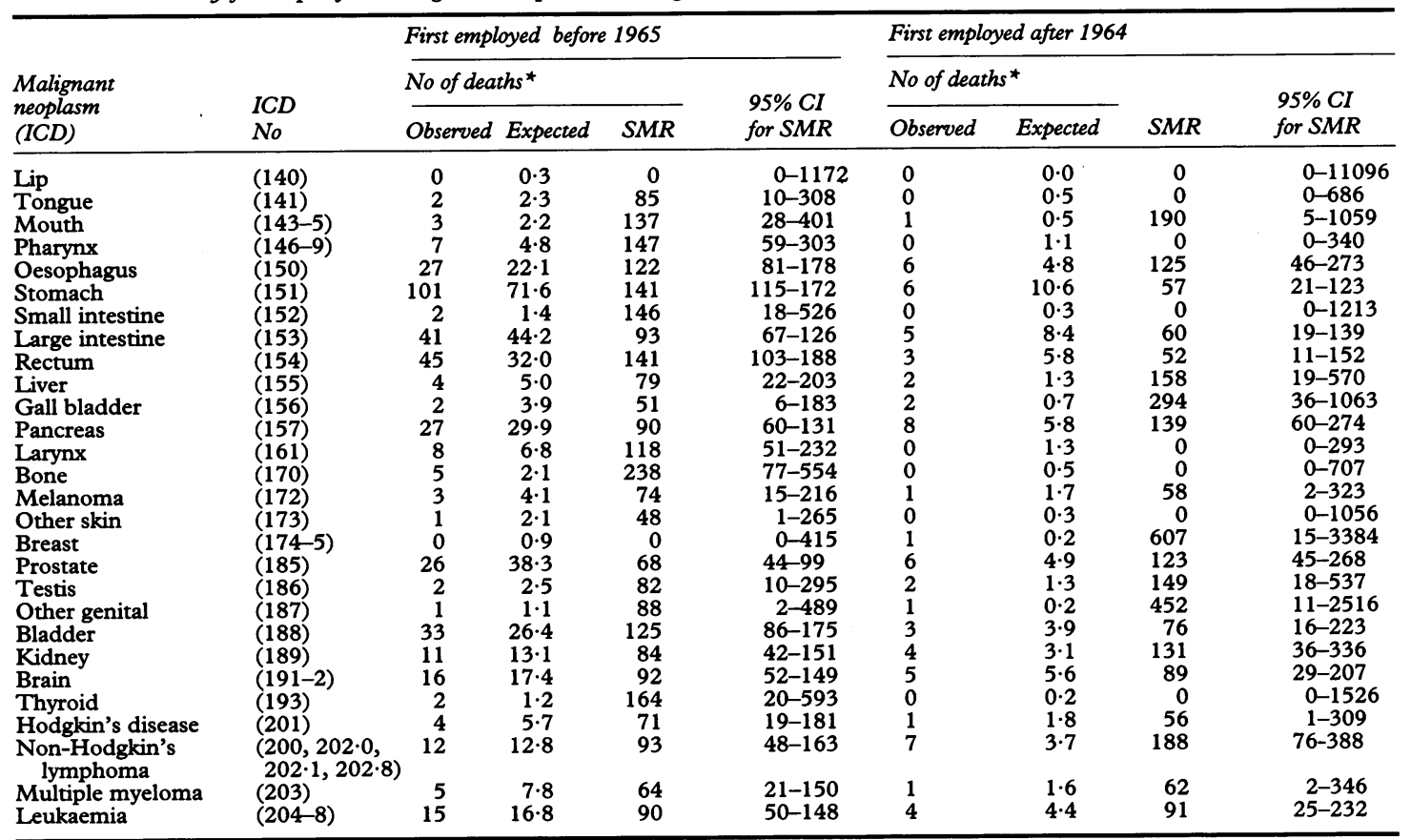

^For explanations see table 2. 
Table 5 Mortality from cancer of the lung during 1941-1989 among men by factory and category of exposure to formaldehyde with adjustment for local rates

\begin{tabular}{|c|c|c|c|c|c|c|c|c|c|}
\hline \multirow{3}{*}{ Factory } & \multirow{3}{*}{$\begin{array}{l}\text { Exposure } \\
\text { category }\end{array}$} & \multicolumn{4}{|c|}{ First employed before 1965} & \multirow{2}{*}{\multicolumn{2}{|c|}{$\begin{array}{l}\text { First employed after } 1964 \\
\text { No of deaths* }\end{array}$}} & \multirow[b]{3}{*}{$S M R$} & \multirow{3}{*}{$\begin{array}{l}95 \% C I \\
\text { for SMR }\end{array}$} \\
\hline & & \multicolumn{2}{|c|}{ No of deaths* } & \multirow[b]{2}{*}{$S M R$} & \multirow{2}{*}{$\begin{array}{l}95 \% C I \\
\text { for SMR }\end{array}$} & & & & \\
\hline & & Observed & Expected & & & Observed & Expected & & \\
\hline Borden & $\begin{array}{l}\text { Nil/background } \\
\text { Low } \\
\text { Moderate } \\
\text { High } \\
\text { Unknown }\end{array}$ & $\begin{array}{l}8 \\
2 \\
1 \\
1 \\
0\end{array}$ & $\begin{array}{l}6 \cdot 5 \\
2 \cdot 0 \\
2 \cdot 6 \\
0 \cdot 5 \\
1 \cdot 7\end{array}$ & $\begin{array}{r}122 \\
99 \\
39 \\
187 \\
0\end{array}$ & $\begin{array}{c}53-241 \\
12-357 \\
1-217 \\
5-1040 \\
0-216\end{array}$ & $\begin{array}{l}9 \\
1 \\
3 \\
1 \\
0\end{array}$ & $\begin{array}{l}5 \cdot 3 \\
1 \cdot 1 \\
1.9 \\
0 \cdot 2 \\
0 \cdot 7\end{array}$ & $\begin{array}{r}171 \\
87 \\
156 \\
413 \\
0\end{array}$ & $\begin{array}{c}78-325 \\
2-487 \\
32-456 \\
11-2300 \\
0-547\end{array}$ \\
\hline $\begin{array}{l}\text { Synthite } \\
\text { (West } \\
\text { Bromwich) }\end{array}$ & $\begin{array}{l}\text { Nil/background } \\
\text { Low } \\
\text { Moderate } \\
\text { High } \\
\text { Unknown }\end{array}$ & $\begin{array}{r}3 \\
11 \\
1 \\
5 \\
1\end{array}$ & $\begin{array}{l}2 \cdot 5 \\
9 \cdot 2 \\
2 \cdot 9 \\
5 \cdot 2 \\
0 \cdot 5\end{array}$ & $\begin{array}{r}122 \\
119 \\
34 \\
96 \\
214\end{array}$ & $\begin{array}{c}25-353 \\
60-214 \\
1-192 \\
31-224 \\
5-1190\end{array}$ & $\begin{array}{l}0 \\
3 \\
0 \\
1 \\
0\end{array}$ & $\begin{array}{l}0.5 \\
2 \cdot 2 \\
0 \cdot 7 \\
2 \cdot 0 \\
0.04\end{array}$ & $\begin{array}{r}0 \\
136 \\
0 \\
50 \\
0\end{array}$ & $\begin{array}{c}0-767 \\
28-398 \\
0-556 \\
1-277 \\
0-9220\end{array}$ \\
\hline $\begin{array}{l}\text { Synthite } \\
\text { (Mold) }\end{array}$ & $\begin{array}{l}\text { Nil/background } \\
\text { Low } \\
\text { Moderate } \\
\text { High } \\
\text { Unknown }\end{array}$ & $\begin{array}{l}1 \\
6 \\
1 \\
1 \\
1\end{array}$ & $\begin{array}{l}2 \cdot 3 \\
3 \cdot 1 \\
0 \cdot 8 \\
1 \cdot 5 \\
0 \cdot 6\end{array}$ & $\begin{array}{r}43 \\
194 \\
129 \\
65 \\
181\end{array}$ & $\begin{array}{c}1-241 \\
71-422 \\
3-721 \\
2-362 \\
5-1010\end{array}$ & $\begin{array}{l}2 \\
0 \\
0 \\
3 \\
0\end{array}$ & $\begin{array}{l}0.9 \\
1 \cdot 1 \\
0 \cdot 8 \\
0 \cdot 5 \\
0 \cdot 3\end{array}$ & $\begin{array}{r}232 \\
0 \\
0 \\
552 \\
0\end{array}$ & $\begin{array}{c}28-839 \\
0-348 \\
0-444 \\
114-1610 \\
0-1130\end{array}$ \\
\hline BIP & $\begin{array}{l}\text { Nil/background } \\
\text { Low } \\
\text { Moderate } \\
\text { High } \\
\text { Unknown }\end{array}$ & $\begin{array}{r}0 \\
17 \\
23 \\
165 \\
11\end{array}$ & $\begin{array}{r}0 \cdot 2 \\
14 \cdot 2 \\
21 \cdot 9 \\
131 \cdot 1 \\
11 \cdot 3\end{array}$ & $\begin{array}{r}0 \\
119 \\
105 \\
126 \\
97\end{array}$ & $\begin{array}{c}0-1990 \\
70-191 \\
67-158 \\
107-147 \\
48-174\end{array}$ & $\begin{array}{r}-1 \\
2 \\
12 \\
0\end{array}$ & $\begin{array}{l}- \\
1 \cdot 2 \\
2 \cdot 2 \\
8 \cdot 7 \\
1 \cdot 3\end{array}$ & $\begin{array}{r}\overline{83} \\
90 \\
138 \\
0\end{array}$ & $\begin{array}{r}- \\
2-460 \\
11-326 \\
71-241 \\
0-279\end{array}$ \\
\hline $\begin{array}{l}\text { Ciba-Geigy } \\
\text { (from) } \\
1958\end{array}$ & $\begin{array}{l}\text { Nil/background } \\
\text { Low } \\
\text { Moderate } \\
\text { High } \\
\text { Unknown }\end{array}$ & $\begin{array}{l}2 \\
7 \\
2 \\
4 \\
0\end{array}$ & $\begin{array}{l}3 \cdot 6 \\
9 \cdot 3 \\
2 \cdot 2 \\
3 \cdot 1 \\
2 \cdot 0\end{array}$ & $\begin{array}{r}57 \\
76 \\
90 \\
127 \\
0\end{array}$ & $\begin{array}{r}7-206 \\
31-157 \\
11-324 \\
35-326 \\
0-189\end{array}$ & $\begin{array}{l}1 \\
6 \\
1 \\
0 \\
0\end{array}$ & $\begin{array}{l}1 \cdot 8 \\
6 \cdot 1 \\
0 \cdot 3 \\
0 \cdot 3 \\
0 \cdot 6\end{array}$ & $\begin{array}{r}55 \\
99 \\
372 \\
0 \\
0\end{array}$ & $\begin{array}{c}1-306 \\
36-215 \\
9-207 \\
0-1070 \\
0-570\end{array}$ \\
\hline BP & $\begin{array}{l}\text { Nil/background } \\
\text { Low } \\
\text { Moderate } \\
\text { High } \\
\text { Unknown }\end{array}$ & $\begin{array}{l}49 \\
25 \\
- \\
0\end{array}$ & $\begin{array}{l}46 \cdot 1 \\
24 \cdot 3 \\
- \\
-0 \cdot 2\end{array}$ & $\begin{array}{c}106 \\
\frac{103}{0}\end{array}$ & $\begin{array}{l}79-140 \\
67-152 \\
- \\
-0-1540\end{array}$ & $\begin{array}{r}6 \\
2 \\
- \\
0\end{array}$ & $\begin{array}{l}4.9 \\
2 \cdot 1 \\
- \\
- \\
0.01\end{array}$ & $\begin{array}{r}122 \\
98 \\
- \\
0\end{array}$ & $\begin{array}{l}45-267 \\
12-352 \\
= \\
\overline{0}-73800\end{array}$ \\
\hline Total & $\begin{array}{l}\text { Nil/background } \\
\text { Low } \\
\text { Moderate } \\
\text { High } \\
\text { Unknown }\end{array}$ & $\begin{array}{r}63 \\
68 \\
28 \\
176 \\
13\end{array}$ & $\begin{array}{r}61 \cdot 2 \\
62 \cdot 1 \\
30 \cdot 4 \\
141 \cdot 5 \\
16 \cdot 2\end{array}$ & $\begin{array}{r}103 \\
109 \\
92 \\
124 \\
80\end{array}$ & $\begin{array}{r}79-132 \\
85-139 \\
61-133 \\
107-144 \\
43-137\end{array}$ & $\begin{array}{r}18 \\
13 \\
6 \\
17 \\
0\end{array}$ & $\begin{array}{r}13.3 \\
13.7 \\
5.9 \\
11.8 \\
3.0\end{array}$ & $\begin{array}{r}135 \\
95 \\
102 \\
144 \\
0\end{array}$ & $\begin{array}{r}80-214 \\
50-162 \\
37-221 \\
84-230 \\
0-122\end{array}$ \\
\hline
\end{tabular}

${ }^{\star}$ For explanation of death rates see table 3.

Table 6 Mortality from cancer of the lung during 1941-1989 among men at BIP in the high category of formaldehyde exposure by length of employment and duration of follow up with adjustment for local rates

\begin{tabular}{|c|c|c|c|c|c|c|c|c|}
\hline \multirow{3}{*}{$\begin{array}{l}\text { Period of } \\
\text { time }\end{array}$} & \multicolumn{4}{|c|}{ First employed before 1965} & \multicolumn{4}{|c|}{ First employed after 1964} \\
\hline & \multicolumn{2}{|c|}{ No of deaths* } & \multirow[b]{2}{*}{$S M R$} & \multirow{2}{*}{$\begin{array}{l}95 \% C I \\
\text { for SMR }\end{array}$} & \multicolumn{2}{|c|}{ No of deaths* } & \multirow[b]{2}{*}{$S M R$} & \multirow{2}{*}{$\begin{array}{l}95 \% C I \\
\text { for } S M R\end{array}$} \\
\hline & Observed & Expected & & & Observed & Expected & & \\
\hline \multicolumn{9}{|c|}{ Years of employment: } \\
\hline 0 & 71 & $52 \cdot 0$ & 137 & $107-172$ & 4 & $3 \cdot 2$ & 125 & $34-320$ \\
\hline $1-4$ & 37 & $31 \cdot 2$ & 119 & $84-164$ & 1 & $1 \cdot 8$ & 55 & $2-309$ \\
\hline $5-9$ & 18 & $12 \cdot 2$ & 147 & $87-233$ & 4 & 1.8 & 217 & 59-557 \\
\hline $10-14$ & 8 & $6 \cdot 6$ & 122 & $53-240$ & 2 & $1 \cdot 3$ & 160 & $19-578$ \\
\hline$\geqslant 15$ & 28 & $25 \cdot 6$ & 109 & $73-158$ & 1 & 0.5 & 214 & $5-1190$ \\
\hline Unknown & 3 & $3 \cdot 3$ & 92 & $19-268$ & 0 & $0 \cdot 1$ & 0 & $0-3920$ \\
\hline \multicolumn{9}{|c|}{ Years of follow up: } \\
\hline $0-4$ & 2 & $3 \cdot 5$ & 58 & $7-209$ & 1 & $1 \cdot 0$ & 98 & $2-543$ \\
\hline $5-9$ & 10 & $6 \cdot 6$ & 151 & $73-278$ & 2 & 1.8 & 109 & $13-394$ \\
\hline $10-14$ & 11 & $11 \cdot 0$ & 100 & $50-179$ & 4 & $2 \cdot 7$ & 150 & $41-385$ \\
\hline $15-19$ & 25 & $16 \cdot 6$ & 151 & $98-223$ & 5 & $\overline{2} \cdot 2$ & 228 & $74-531$ \\
\hline $20-24$ & 29 & $22 \cdot 6$ & 128 & $86-184$ & 0 & 0.9 & 0 & $0-391$ \\
\hline$\geqslant 25$ & 88 & $70 \cdot 4$ & 125 & $100-154$ & - & - & - & - \\
\hline
\end{tabular}

For explanation of death rates see table 3 . 
Table 7 Mortality from cancer of the lung during 1941-1989 among men entering employment at BIP before 1965 by cumulative dose

\begin{tabular}{|c|c|c|c|c|}
\hline \multirow{2}{*}{$\begin{array}{l}\text { Cumulative } \\
\text { dose group }\end{array}$} & \multicolumn{2}{|c|}{ No of deaths* } & \multirow[b]{2}{*}{$S M R$} & \multirow{2}{*}{$\begin{array}{l}95 \% C I \\
\text { for } S M R\end{array}$} \\
\hline & Observed & Expected & & \\
\hline \multicolumn{5}{|l|}{ Analysis At } \\
\hline $\begin{array}{c}0-4 \\
5-19 \\
20-99 \\
\geqslant 100 \\
\text { Analysis Bł }\end{array}$ & $\begin{array}{l}42 \\
46 \\
47 \\
59\end{array}$ & $\begin{array}{l}31 \cdot 6 \\
34 \cdot 3 \\
42 \cdot 2 \\
45 \cdot 6\end{array}$ & $\begin{array}{l}133 \\
134 \\
111 \\
129\end{array}$ & $\begin{array}{l}96-180 \\
98-179 \\
82-148 \\
98-167\end{array}$ \\
\hline $\begin{array}{c}0-10 \\
11-40 \\
41-200 \\
\geqslant 201\end{array}$ & $\begin{array}{l}60 \\
43 \\
47 \\
32\end{array}$ & $\begin{array}{l}40 \cdot 0 \\
33 \cdot 8 \\
39 \cdot 9 \\
26 \cdot 7\end{array}$ & $\begin{array}{l}150 \\
127 \\
118 \\
120\end{array}$ & $\begin{array}{r}115-193 \\
92-171 \\
87-157 \\
82-169\end{array}$ \\
\hline
\end{tabular}

*For explanation of death rates see table 3.

†Analysis A: cumulative dose on a continual basis with subjectively estimated concentrations; mortality analysis continual from date of entry to the end of 1989 .

¥Analysis B: cumulative dose to 1960 with scoring system; mortality analysis from 1961 to the end of 1989 .

Table 8 Mortality from cancer of the lung during 1941-89 among men at BIP in the high category of formaldehyde exposure by calendar period of first employment with adjustment for local rates

\begin{tabular}{|c|c|c|c|c|}
\hline \multirow{2}{*}{$\begin{array}{l}\text { Period of } \\
\text { first } \\
\text { employment }\end{array}$} & \multicolumn{2}{|c|}{ No of deaths* } & \multirow[b]{2}{*}{$S M R$} & \multirow{2}{*}{$\begin{array}{l}95 \% C I \\
\text { for } S M R\end{array}$} \\
\hline & Observed & Expected & & \\
\hline $\begin{array}{l}1936-45 \\
1946-55 \\
1956-64 \\
1965-82\end{array}$ & $\begin{array}{l}34 \\
88 \\
43 \\
12\end{array}$ & $\begin{array}{r}22 \cdot 1 \\
76 \cdot 9 \\
32 \cdot 1 \\
8 \cdot 7\end{array}$ & $\begin{array}{l}154 \\
114 \\
134 \\
138\end{array}$ & $\begin{array}{r}107-215 \\
92-141 \\
97-181 \\
71-241\end{array}$ \\
\hline
\end{tabular}

${ }^{\star}$ For explanation of death rates see table 3 .

Further analyses with, for example, different time periods similarly showed no relation.

Table 8 gives findings for lung cancer among men classified as exposed to high concentrations of formaldehyde at the BIP factory by their calendar period of first employment. Mortality has been slightly higher among men who entered up to 1945 than subsequently.

\section{Discussion}

The original impetus for this study was the suggestion from animal experiments that formaldehyde may be carcinogenic to the nasal cavity and sinuses in humans. Only one of the 3201 deaths reported in this paper, however, was certified as nasal cancer, compared with a total of 1.7 expected from national death rates. The one death occurred during the extended follow up period and was a man exposed for five years to formaldehyde in the low exposure group $(0 \cdot 1-0.5 \mathrm{ppm}) 37$ years after his start of employment. Nasal cancer has not been mentioned as a contributory cause of death on any of the other 3201 death certificates, nor have any non-fatal cases of nasal cancer been reported from the national cancer registry. Among 26561 industrial workers in the similar large study (4396 deaths) from the United States ${ }^{8}$ there was also no excess of nasal cancer with two observed deaths compared with $2 \cdot 8$ expected. In other studies of industrial and professional workers and of nonoccupational exposure there has been inconsistent evidence of a formaldehyde related risk of nasal cancer, with overall a small non-significant relative risk of 1.1 unrelated to duration or amount of exposure. ${ }^{9}$ The statistical power of the cohort studies, including this one, to detect a modest increase in nasal cancer-a rare disease with typically more than 20 years between first exposure and diagnosis-is still less than desired (this study alone, for example, has only $54 \%$ power to detect a relative risk of 4 starting from 20 years after first exposure).

Experiments in animals did not show an increase in lung cancer from exposure to formaldehyde, but in humans the bronchus is a credible target organ as inhalation is the primary route of exposure. In this study for men employed before 1965 there was an excess of lung cancer overall among formaldehyde workers of $23 \%$ when compared with death rates for men in England and Wales, but this decreased to $12 \%$ when adjusted for mortality from lung cancer in the areas of the factories. The reasons for preferring the last comparison in this study have been detailed earlier ${ }^{714}$ but basically relate to most of the workforce being local men judged by places of birth, employment, and death. 
Nevertheless, even with the reduction, the overall excess is statistically significant and particularly relates to the factory (BIP) where more men were classified as having high exposure to formaldehyde. Within this factory and overall there are small suggestions of increasing mortality from lung cancer with increasing job categorisation of exposure to formaldehyde. Also workers employed in earlier years when exposures were probably greater tend to have higher death rates from lung cancer. Within the high exposure group, however, there is no relation of mortality to duration of employment or to time since starting exposure. Also, there is no apparent relation of lung cancer to cumulative exposure among this workforce. For men first employed in any one of the factories after 1964 the SMR from lung cancer overall (113) is similar to that of the earlier workers (112).

This excess of lung cancer overall is comparable with that $(10 \%)$ found among industrial workers in the United States. ${ }^{8}$ In that study there were also no consistent indications of lung cancer being associated with intensity or duration of exposure to formaldehyde nor with cumulative exposure when looked at in various ways. In neither of these studies was information on smoking habits available ${ }^{815}$ and although this is a limitation there is little suggestion of smoking related excesses or deficits-for example, mortality from respiratory disease is similar to that expected. Among the other studies reviewed by Blair and colleagues ${ }^{9}$ there were fewer with more lung cancer than with less, although a number were of professional workers in whom smoking habits were probably lower. Overall the evidence is not yet clear and the lung cancer rates from this present follow up are higher than those reported previously up to 1981 .

The buccal cavity and pharynx are also potential targets for exposure from inhalation of formaldehyde in humans. In this study overall there have been 13 observed deaths compared with 11.8 expected (observed/expected $=1 \cdot 10,95 \%$ CI $0 \cdot 59-1 \cdot 89$ ). For specific subsites there were no indications of excesses including for the pharynx (seven observed, 5.9 expected). Within the pharynx in particular there were no deaths recorded for cancer of the nasopharynx (expected number estimated at 1.3). There have not been any deaths where nasopharyngeal cancer has been mentioned on the death certificate as a contributory cause, nor any non-fatal cases notified from the National Cancer Registry. This contrasts with the findings of Blair and colleagues ${ }^{8}$ who reported seven cases compared with $2 \cdot 2$ expected, but in aggregate among all published studies there is only a small relative excess of cancer of the nasopharynx. ${ }^{9}$

In terms of mortality from all causes of death the level has risen among those first employed before
1965 from an SMR of 87 reported earlier up to the end of $1981^{7}$ to an SMR of 103 when the follow up was extended to the end of 1989 . This is consistent with the wearing off of any healthy worker effect, but is also at least partly due to the extensive checking of the status of cohort members before the present analysis. Thus we can estimate that the earlier reported SMR would have been nearer 100 than to the value of 87 published as a consequence of the revision of the database. This effect was seen similarly across separate causes of death, but was slightly greater at BIP than the other factories. Among men first employed after 1964 the SMR was 95.

For all cancers combined there is an excess of $14 \%$ compared with rates in England and Wales in men first employed before 1965 and a deficit of 3\% among those joining the workforce subsequently. After allowing for the $23 \%$ raised lung cancer rates in the first group, there were 454 deaths from all other cancers compared with 421 expected (observed/expected $=1 \cdot 08,95 \%$ CI 0.98-1.18). As can be seen from table 4 the main contribution to this SMR of 108 (33 excess deaths) was cancer of the stomach for which there was an SMR of 141 (29 excess deaths). This increase in stomach cancer reduced to an SMR of 124 (95\% CI 101-151) when adjusted for local mortality and some excess was seen consistently across all the six factories. There was no indication of a relation of cancer of the stomach with formaldehyde exposure category, duration of employment, or time since first employment. There was no suggestion of an excess of stomach cancer among men first employed after 1964 (table 4, SMR 57), or in the study from the United States (SMR 83), nor has stomach cancer been indicated as raised in other studies. ${ }^{9}$ For rectal cancer, the other site with a statistically significant excess, the SMR of 141 reduced after adjustment for local mortality levels to 113 (95\% CI 82-151) and showed no indications of a relation with formaldehyde in this or other studies. ${ }^{9}$ Other cancers that had shown excesses in some other studies-such as cancers of colon (large intestine), skin, prostate, and brain as well as Hodgkin's disease and leukaemia - are not raised in this study, whereas bladder cancer shows a non-significant increase.

For diseases of the respiratory system there is an excess of $15 \%$ overall compared to rates in England and Wales for workers first employed before 1965, which reduces to $5 \%$ when adjustments are made for local mortality. The increases are larger for the BIP factory at $42 \%$ and $17 \%$ respectively. As for the specific cancers mentioned earlier there is no indication of this excess being related to formaldehyde exposure. The increased respiratory disease among the BIP workers is of a similar magnitude to that for cancer of the lung and, with the same lack of relation to formaldehyde exposure, may indicate 
higher cigarette smoking or some other environmental pollutant among these employees.

The following chemicals, among others, are known to have been used at BIP during the period covered by the study. Exposure to these was dependent on department and date. Asbestos was used as a filler in moulding materials produced from the time of the second world war to about 1967. The types of asbestos used were chrysotile (calcined at $600^{\circ} \mathrm{C}$ ) and anthophyllite. Scandinavian spruce woodflour was used as a filler from the late 1930s until 1979. Epichlorhydrin was used in the resins department since 1976. Tris (2, 3-dibromopropyl) phosphate was used in 1977 for a few months in the resins department only. Lead chrome pigments containing hexavalent chromium had been used in one department since 1972.

There was a greater percentage of British workers assigned to the "high" exposure category (qualitatively estimated at over $2 \mathrm{ppm}$ ) in the study ( $35 \%$ among those first employed before 1965 and $21 \%$ among those first employed after 1964) compared with the study in the United States (3\% overall). This can probably be ascribed to two factors. Firstly, most of the British cohort worked at BIP where the enclosed structure of the factor building in the early period being studied would have been taken into account when the subjective assessment of conditions was being made. Results of air monitoring carried out in the departments concerned before the improvements in working conditions were made during the mid-1970s would seem to support this assessment. Secondly, when jobs were being placed into qualitative categories of exposure in the British study, some disagreement occurred as to which of two adjacent grades was most appropriate-for example, high or moderate? To achieve consistency across all the factories the higher of the two was always used. It is not clear how differences were resolved in the United States study. ${ }^{16}$ Also, as mentioned earlier, if a man in our study had more than one job he was classified to that considered to have the higher exposure.

In summary, this study with one nasal cancer death compared with 1.7 expected does not support the original evidence from animals on carcinogenicity. This could be, among other reasons, because of the lower exposures that were experienced and could be tolerated by humans or because formaldehyde is not a (nasal) carcinogen in humans. We have found no cases of nasopharyngeal cancer compared to about 1.3 expected which lends no weight to the suggestion of a relation with formaldehyde exposure in a comparable study in the United States. For lung cancer, respiratory disease, and stomach cancer there are slight excesses overall but no relation with estimated cumulative dose or time since first exposure. The results of this and other published studies do not yet justify a firm conclusion about the human carcinogenicity or otherwise of formaldehyde but would suggest that at past, and more so therefore at current, levels of exposure there can be no more than a very weak effect.

We are grateful for the cooperation of the companies involved in this study and for the help of their staff. We acknowledge the roles of the National Health Service Central Register in Southport and the Department of Social Security in Newcastle in enabling us to follow up the workforces. The study was approved by the British Medical Association ethics committee. We thank many colleagues for their help with the study, in particular Caroline Powell and Tony Fletcher for data organisation and Gill Strange for preparing the manuscript. Finally we acknowledge the interest and help of the Chemical Industries Association Formaldehyde Health Impact Study Team.

1 International Agency for Research on Cancer. IARC Monographs on the evaluation of carcinogenic risks to humans: some industrial chemicals and dyestuffs. Vol 29. Lyon: IARC, 1982: 345.

2 National Research Council. Formaldehyde: an assessment of its health effects. Washington: National Academy of Sciences, 1982.

3 Hendrick DJ, Lane DJ. Occupational formalin asthma. $\mathrm{Br} \mathscr{B}$ Ind Med 1977;34:11.

4 Auerbach C, Moutschen-Dahmen M, Moutschen J. Genetic and cytogenetical effects of formaldehyde and related cone pounds. Mutat Res 1977;39:317-62.

5 Swenberg JA, Kerns WD, Mitchell RI, Gralla EJ, Pavkov KI. Induction of squamous cell carcinomas of the rat nasal cavity by inhalation exposure to formaldehyde vapor. Cancer Res 1980;40:3398-401.

6 Albert RE, Sellukmar AR, Laskin S, Kuschner M, Nelson N, Snyder CA. Gaseous formaldehyde and hydrogen chloride induction of nasal cancer in the rat. $\mathcal{F}$ Nat Cancer Inst 1982 68:597-603.

7 Acheson ED, Barnes HR, Gardner MJ, Osmond C, Pannett $B$, Taylor CP. Formaldehyde in the British chemical industry: an occupational cohort study. Lancet 1984;i:61 1-6.

8 Blair A, Stewart P, O'Berg M, Gaffey W, Walrath J, Ward I, et al. Mortality among industrial workers exposed to formaldehyde. F Nat Cancer Inst 1986;76:1071-84.

9 Blair A, Saracci R, Stewart PA, Hayes RB, Shy C. Epidemiologic evidence on the relationship between formaldehyde exposure and cancer. Scand $\mathcal{f}$ Work Environ Health 1990;16:381-93.

10 World Health Organisation. International Classification of Diseases. 9th rev. Geneva: WHO, 1977

11 Gardner MJ, Winter PD, Taylor CP, Acheson ED. Atlas of cancer mortality in England and Wales, 1968-1978. Chichester: Wiley, 1983.

12 Office of Population Censuses and Surveys. Mortality statistics by area, 1980-89. London: HMSO, 1982-91. (Series DH5 Nos 7-16.)

13 Acheson ED, Barnes HR, Gardner MJ, Osmond C, Pannett B, Taylor CP. Formaldehyde process workers and lung cancer. Lancet 1984:i:1066-7.

14 Gardner MJ, Osmond C, Pannett B, Acheson ED. $\omega$ Formaldehyde, lung cancer, and bronchitis. Lancet 1986;i: 437-8.

15 Acheson ED, Barnes HR, Gardner MJ, Osmond C, Pannett B, Taylor CP. Cohort study of formaldehyde process workers. Lancet $1984 ;$ ii: 403 .

16 Stewart PA, Blair A, Cubit DA, Bales RE, Kaplan SA, Ward J, et al. Estimating historical exposures to formaldehyde in a retrospective mortality study. Applied Industrial Hygiene 1986:1:34-41.

Accepted 14 December 1992 